\title{
Configurações
}

Revista Ciências Sociais

$27 \mid 2021$

Vária

Gênero e desigualdade na academia brasileira: uma análise a partir dos bolsistas de produtividade em pesquisa do $\mathrm{CNPq}$

Gender and inequality in the Brazilian academia: an analysis from $\mathrm{CNPq}$

research productivity fellows

Genre et inégalité au sein de l'académie brésilienne : une analyse des boursiers

de productivité de la recherche du CNPq

Amurabi Oliveira, Marina Félix de Melo, Quemuel Baruque de Rodrigues e Mayres Pequeno

OpenEdition

Journals

Edição electrónica

URL: https://journals.openedition.org/configuracoes/11979

DOI: 10.4000/configuracoes. 11979

ISSN: 2182-7419

Editora

Centro de Investigação em Ciências Sociais

Edição impressa

Paginação: 75-93

ISSN: 1646-5075

\section{Refêrencia eletrónica}

Amurabi Oliveira, Marina Félix de Melo, Quemuel Baruque de Rodrigues e Mayres Pequeno, «Gênero e desigualdade na academia brasileira: uma análise a partir dos bolsistas de produtividade em pesquisa do CNPq», Configurações [Online], 27 | 2021, posto online no dia 24 junho 2021, consultado o 26 junho 2021. URL: http://journals.openedition.org/configuracoes/11979 ; DOI: https://doi.org/10.4000/ configuracoes. 11979 
Oliveira, Amurabi; Melo, Marina Félix de; Rodrigues, Quemuel Baruque de; Pequeno, Mayres - Gênero e desigualdade na academia Brasileira: uma análise a partir dos bolsistas de produtividade em pesquisa do CNPq. Configurações, vol. 27, 2021, pp. 75-93.

\title{
Gênero e desigualdade na academia brasileira: uma análise a partir dos bolsistas de produtividade em pesquisa do CNPq
}

\author{
AMURABI OLIVEIRA* \\ Universidade Federal de Santa Catarina, Brasil \\ MARINA FÉLIX DE MELO** \\ Universidade Federal de Alagoas, Brasil \\ QUEMUEL BARUQUE DE RODRIGUES*** \\ Universidade Federal de Pernambuco, Brasil \\ MAYRES PEQUENO**** \\ Universidade Federal de Alagoas, Brasil
}

\begin{abstract}
Resumo
O presente artigo discute as desigualdades de gênero no campo acadêmico. Através de uma abordagem quanti-qualitativa, analisa-se a distribuição das bolsas de produtividade em pesquisa do CN Pq no Brasil em relação a homens e mulheres. Conclui-se que essas desigualdades são aprofundadas pela menor presença das mulheres entre os bolsistas nos níveis mais altos, bem como entre as áreas que recebem mais recursos. Verifica-se ainda que não existe diferença estatisticamente significativa entre homens e mulheres no que se refere a um índice de produtividade acadêmica criado neste trabalho como referência empírica à temática discutida sobre produção científica e gênero, tampouco em relação à quantidade de publicações qualificadas entre eles e elas.
\end{abstract}

Palavras-chave: campo acadêmico, desigualdade de gênero, CN Pq, Universidade.

\footnotetext{
Abstract

Gender and inequality in the Brazilian academia: an analysis from CNPq research productivity fellows

This article discusses gender inequalities in the academic field. Through a quantitative and qualitative approach, the distribution of CNPq research productivity grants in Brazil in relation to men and women is analysed. It is concluded that these inequalities are deepened by the lower presence of women among the scholarship holders at the highest levels, as well as in the areas that receive more resources. It is also verified that there is no statistically significant difference between men and women with regard to 
an academic productivity index created for this investigation as an empirical reference for the topic discussed regarding scientific production and gender, nor in relation to the quantity of qualified publications between men and women.

Keywords: academic field, gender inequality, CN Pq, University.

\section{Resumé \\ Genre et inégalité au sein de l'académie brésilienne : une analyse des boursiers de productivité de la recherche du CNPq}

Cet article traite des inégalités entre les sexes dans le domaine universitaire. La répartition des subventions de productivité de la recherche du CN Pq au Brésil par rapport aux hommes et aux femmes est analysée grâce à une approche quantitative et qualitative. Nous concluons que ces inégalités sont aggravées par la moindre présence des femmes parmi les boursiers aux plus hauts niveaux, ainsi que parmi les régions qui reçoivent le plus de ressources. Il est également vérifié qu'il n'y a pas de différence statistiquement significative entre les hommes et les femmes en ce qui concerne un indice de productivité académique créé dans ce travail comme référence empirique au sujet abordé sur la production scientifique et le genre, ni par rapport à la quantité de publications qualifiées entre eux et elles.

Mots-clés: domaine académique, inégalité des genres, CN Pq, Université.

\section{Introdução}

A relação entre a produção e a reprodução das desigualdades sociais e os sistemas educativos tem sido o cerne do debate no campo da Sociologia da Educação nas últimas décadas. No caso brasileiro nota-se um especial destaque se inserirmos esse debate no âmbito das discussões sobre as profundas desigualdades de classe, raça e gênero (Barbosa e Gadin, 2020; Oliveira e Silva, 2020). Este problema mostra-se cada vez mais complexo ao considerarmos, por exemplo, a crescente participação feminina no ensino superior em diversas partes do mundo, e seu êxito nos mais diferentes níveis (Marrero, 2006). Ao mesmo tempo, contrasta com a reprodução das desigualdades de gênero na academia, o que tem sido debatido por uma extensa literatura sobre o tema (Toren, 1993; Olsen, Maple e Stage, 1995; Poole, Bornholot e Summers, 1997; Probert, 2005; Ogbogu, 2011; Grossi et al., 2016; Sarsons, 2017; Weisshaar, 2017)1.

No caso brasileiro, podemos indicar que tem havido um crescente interesse no campo educacional em discutir temas relacionados a gênero, ainda que

1 Recomenda-se consulta em periódicos especializados às temáticas de gênero e trabalho doméstico/ familiar a exemplo de Studies in Higher Education; Research in Higher Education; Higher Education; The Journal of Higher Education. 
predominem os estudos voltados para a educação básica (Ferreira e Coronel, 2017). Em período mais recente, no entanto, tem havido um notório interesse também na pesquisa sobre as desigualdades de gênero no campo acadêmico (Lopes, 1998; Lima e Costa, 2016; Freitas e Luz, 2017). De forma mais específica, dada a centralidade que a Bolsa de Produtividade em Pesquisa (PQ) do Conselho Nacional de Desenvolvimento Científico e Tecnológico (CNPq) assume no processo de legitimação das hierarquias acadêmicas no Brasil, a reflexão sobre a distribuição heterogênea desse tipo de bolsa, considerando-se a questão do gênero, tem chamado a atenção também de diversos pesquisadores (Guedes, Azevedo e Ferreira, 2015; Barros e Silva, 2019).

O objetivo central da presente investigação é o de examinar a distribuição das bolsas de produtividade em pesquisa do $\mathrm{CNPq}$ no Brasil em relação a homens e mulheres. Sustentamos como hipótese experimental que, embora homens e mulheres não tenham diferenças significativas em relação à produção acadêmica e científica, os acessos às bolsas e ao topo da carreira acadêmica no Brasil são mais restritos às mulheres que aos homens, sendo o sexo masculino dominante no campo científico de reconhecimento. A contribuir para essa discussão, buscamos avançar no debate através de uma análise quanti-qualitativa, pela qual construímos e analisamos um índice de produtividade acadêmica, que ultrapassa a dimensão descritiva da desigual distribuição de recursos materiais e simbólicos no campo acadêmico entre homens e mulheres, sintetizada aqui através das bolsas PQ. Também como objetivo específico, buscamos observar possíveis diferenças estatisticamente significativas em distintas variáveis da produção acadêmica de homens e mulheres, considerando as várias áreas do conhecimento às quais se vinculam os pesquisadores do $\mathrm{CN} \mathrm{Pq}$ e suas diferentes regiões de produção científica.

Observa-se que nosso objetivo não se volta para a explicação das desigualdades de gênero na academia, o que fugiria ao foco e escopo de nosso artigo. Os dados que trazemos aqui produzem um retrato da desigualdade de gênero na academia brasileira, o que é analisado a partir de uma abordagem quantitativa das posições mais elevadas do campo acadêmico brasileiro. Como se trata de uma aproximação inicial com esse cenário, almeja-se aprofundar a discussão em trabalhos posteriores, nos quais tentaremos mais incisivamente explicar os fundamentos dessas desigualdades. Em todo o caso, compreendemos que a apresentação desses dados tem um grande valor para pesquisas futuras - nossas e de outros pesquisadores e outras pesquisadoras - que busquem compreender a dinâmica da desigualdade na academia brasileira.

Para uma melhor compreensão por parte de nossos leitores acerca da discussão que pretendemos fomentar aqui, primeiro realizaremos uma apresentação 
mais geral sobre o significado da bolsa de produtividade em pesquisa do CN Pq no campo acadêmico brasileiro, assim como as questões que já foram apontadas anteriormente sobre a desigualdade de gênero nesse âmbito. Em sequência, explanaremos brevemente os procedimentos metodológicos da pesquisa que seguiremos para a apresentação dos resultados.

\section{As bolsas de produtividade em pesquisa do CNPq na hierarquia acadêmica brasileira}

O sistema universitário brasileiro iniciou-se relativamente tarde em comparação a outros países latino-americanos. As primeiras experiências de cursos superiores no País ocorrem apenas no século XIX, e a formação das primeiras universidades somente no século $X^{2}$. No decorrer do século $X X$, observou-se uma paulatina expansão do ensino superior brasileiro, o que foi acompanhado também de uma progressiva diversificação na oferta. As questões de acesso e de equidade se colocaram como um dos principais desafios para o ensino superior brasileiro no século XX (Neves, Raizer, Fachinetto, 2007; Barbosa, 2014, 2015).

Em meio a esse processo, é importante ressaltar que a formação e consolidação de uma comunidade científica no Brasil dependeu não apenas da expansão do ensino superior. Também como fator determinante deste processo, esteve a existência de uma política de formação de quadros e de criação de instituições de fomento à pesquisa, bem como a criação de sociedades científicas. Como bem ressalta Martins (2018: 12):

A criação da Sociedade Brasileira para o Progresso da Ciência (SBPC), em 1948, expressava o crescimento da atividade científica no país e exerceria, a partir de então, uma importante função na organização dos interesses do campo científico. (...) Pouco tempo depois, em 1951, ocorreria a criação de dois organismos que constituiriam um divisor de águas no processo de institucionalização da pesquisa no país: o Conselho Nacional de Pesquisas, CNPq (atualmente Conselho Nacional de Desenvolvimento Científico e Tecnológico), e a Campanha de Aperfeiçoamento de Pessoal Nivel Superior, a CA PES (atualmente Coordenação de Aperfeiçoamento de Pessoal de Nivel Superior). O CN Pq, que posteriormente desempenharia um papel fundamental na construção da pós-graduação nacional, foi criado a partir da convergência

2 Fugiria ao foco e ao escopo deste trabalho realizar uma síntese história do ensino superior brasileiro. Para um maior aprofundamento neste debate, vide Schwartzman (1979). 
de interesses de técnicos do governo e militares e do segmento da comunidade científica nacional.

Todos esses acontecimentos devem ser entendidos como elementos relevantes para a compreensão da formação do campo acadêmico-científico brasileiro. A partir daí são lançadas as bases para a consolidação da pós-graduação nacional, que vem a ganhar um perfil mais semelhante ao atual a partir da Reforma Universitária de 1968. É no contexto da emergência dos primeiros programas de pós-graduação no Brasil que surge, em 1976, a bolsa PQ do $\mathrm{CNPq}$, que acabou se convertendo em um dos principais elementos distintivos e de hierarquização do campo acadêmico brasileiro.

É importante ressaltar que as bolsas PQ são ao mesmo tempo um elemento de distribuição de recursos materiais, considerando que se constituem em um tipo de financiamento à pesquisa e que possibilitam o acesso a outras formas de fomento. As bolsas PQ também articulam recursos simbólicos, uma vez que o status de bolsista PQ possibilita o acesso a determinados comitês científicos e de assessoramento restritos; além disso, esses bolsistas são os avaliadores dos demais pedidos de financiamento realizados ao $\mathrm{CNPq}$, como o pedido de novos bolsistas PQ, editais de financiamento de pesquisa, bolsas de doutorado pleno e sanduíche para o exterior, pós-doutorado, etc.

As bolsas PQ passaram por inúmeras alterações nos níveis e nos valores monetários ao longo dos anos. Atualmente, as bolsas são divididas e hierarquizadas em três categorias: Sênior (PQ-SR); PQ-1 (sendo a categoria 1 subdividida nos níveis 1A, 1B, 1C e 1D); e PQ-2. Apesar da existência dos pré-requisitos gerais estabelecidos pelo $\mathrm{CNPq}$, há ainda os critérios de qualificação definidos pelos Comitês de Assessoramento (CA) de cada área ou pelo Conselho Deliberativo - CD do CN Pq, no caso de Pesquisador Sênior. Tais bolsas são requisitadas individualmente pelos pesquisadores e possuem distintas durações, sendo 36 meses para a nível 2, 48 meses para as bolsas nível 1, e 60 meses para as bolsas sênior. Os pesquisadores nível 1 também recebem, além da bolsa, um auxílio-banca, que visa ajudá-los no financiamento de pesquisas, participação em eventos científicos, processos de editoração de suas publicações, etc.

Os requisitos mínimos comuns a todas as áreas para obtenção da bolsa PQ são: ao menos três anos de doutorado completo por ocasião da implementação da bolsa, para a bolsa nível 2, e oito anos para a bolsa nível 1; o pesquisador deverá se dedicar às atividades constantes em seu pedido de bolsa, podendo o mesmo ser aposentado, desde que mantenha atividades acadêmico-científicas oficialmente vinculadas a instituições de pesquisa e ensino. Ainda segundo o CN Pq, os critérios, independentemente do CA, deverão contemplar os seguintes itens: a) produção científica do candidato; b) formação de recursos humanos em nível de Pós-Graduação; c) contribuição científica e tecnológica e para 
inovação; d) coordenação ou participação principal em projetos de pesquisa; e) participação em atividades editoriais e de gestão científica e administração de instituições e núcleos de excelência científica e tecnológica (CN PQ, 2015).

A considerar tais critérios, torna-se evidente que a atribuição da bolsa PQ a um determinado pesquisador no Brasil significa o reconhecimento da comunidade de pares de que este integra uma "elite acadêmica". Isso fica ainda mais evidente nos níveis mais elevados, uma vez que o CNPQ afirma:

O nível A é reservado a candidatos que tenham mostrado excelência continuada na produção científica e na formação de recursos humanos, e que liderem grupos de pesquisa consolidados. O perfil deste nível de pesquisador deve, na maior parte dos casos, extrapolar os aspectos unicamente de produtividade para incluir aspectos adicionais que mostrem uma significativa liderança dentro da sua área de pesquisa no Brasil e capacidade de explorar novas fronteiras científicas em projetos de risco. (CN PQ, 2015: 2)

Como já indicado, os CA possuem autonomia para estabelecer os critérios para concessão das bolsas PQ. É possível que haja maior ou menor afinidade com os critérios estabelecidos pela CAPES para a avaliação da produção acadêmica, sintetizado pela classificação do qualis da CAPES. Todavia, considerando que majoritariamente os bolsistas PQ estão inseridos em programas de pós-graduação, que por sua vez são avaliados pela CAPES, pode-se inferir que a publicação em revistas bem avaliadas no qualis seja um critério relevante para as diversas áreas, ainda que tenham ganhado espaço nos últimos anos as métricas internacionais, especialmente o fator impacto ${ }^{3}$.

Essa autonomia dos CA também se reverbera em diferentes orientações com relação à forma como concedem tais bolsas. Como indicam Weiner e Vieira (2013: 73-74):

As grandes áreas devem ponderar se as práticas que estão seguindo para avaliar seus pesquisadores estão de acordo com os seus objetivos para as bolsas de produtividade. Obviamente, não existe um objetivo "certo" ou único para a atribuição de bolsas e, portanto, não deve existir uma única métrica que deva ser seguida. No entanto, nos parece que há duas grandes vertentes

3 Sabemos que o sistema qualis da CAPES, que estratifica as revistas científicas por áreas, encontra-se atualmente em revisão, havendo a possibilidade de ser elaborado um qualis unificado para as diversas áreas do conhecimento, assim como sua posterior eliminação, substituindo-o pelas métricas internacionais (fator de impacto e índice h5). Entretanto, os atuais bolsistas orientaram sua produção acadêmica em um cenário no qual o qualis constitui o principal sistema de classificação da produção acadêmica nacional, sendo utilizado inclusive como critério nas provas de admissão para a carreira docente e progressão funcional de muitas universidades. 
para explicar quais são os objetivos de uma bolsa de produtividade, que, se não são contraditórios entre si, são pelo menos diferentes. $O$ primeiro objetivo possível é premiar cientistas de qualidade. O segundo objetivo possível é incentivar a produção de qualidade dos cientistas brasileiros. Há uma diferença importante entre estes dois objetivos: se o objetivo é premiar os cientistas pela qualidade e importância do seu trabalho, então, a história passada do pesquisador é o fator mais importante. Caso o objetivo seja incentivar a produção de qualidade e relevância, então, o futuro deste pesquisador é mais importante que seu passado. É claro que, nesta segunda alternativa, o passado é importante, mas apenas como ferramenta para prever o futuro do pesquisador - na falta de melhores dados acredita-se que o pesquisador, no futuro, terá os mesmos resultados (do ponto de vista de produção científica) que no passado ou, pelo menos, no passado recente. Além do mais, as duas vertentes de objetivos têm impacto muito diferente no agraciado. Quem recebe um prêmio, não precisa mais continuar fazendo o que fez para ganhar o prêmio, apenas quando outra pessoa tiver acum ulado história suficiente que o prêmio será transferido. Quem recebe um incentivo, deve manter pelo menos a mesma produção que fez com que recebesse o incentivo, pois, senão, ele será retirado.

No contexto dessas diferentes orientações existentes nas distintas áreas encontraremos cenários também bastante heterogêneos com relação à distribuição das bolsas por sexo, ainda que essa seja uma realidade que tem se dinamizado nos últimos anos. Como apontam Guedes, Azevedo e Ferreira (2015: 396-397):

Ressalta em primeiro lugar o peso diferenciado de cada uma quanto ao montante total de $P Q$ s, sendo as áreas mais contempladas as das CIÊNCIAS EXATAS E DA TERRA e ENGENHARIAS. A concentração de PQs nessas áreas provoca uma desproporção em geral na presença de mulheres entre os bolsistas produtividade, uma vez que é justamente ali que se reproduz historicamente o fenômeno da baixa representação feminina. As exceções a essa tendência histórica nessas áreas com predomínio masculino, como Química, Engenharia Sanitária e Engenharia Química, constituem casos que merecem investigação específica a respeito das dinâmicas institucionais recentes que propiciaram a alteração desse quadro, com a elevação do número de bolsistas mulheres.

O utro caso de mudança é o das CIÊNCIAS AGRÁRIAS, campo tradicionalmente masculino, em cujo movimento de expansão de $67 \%$ no número de PQs no período 2001-2012 constatamos sinais inovadores de abertura ao ingresso de pesquisadoras. 
Alterações importantes também foram verificadas nas CIÊNCIAS BIOLÓGICAS e nas CIÊNCIAS DA SAÚDE, áreas com patamares mais equânimes e nas quais é franca a tendência de crescimento na proporção de bolsistas mulheres. Já nas CIÊNCIAS HUMANAS e em LINGUÍSTICA, LETRAS E ARTES houve, com algumas exceções, diminuição da histórica predominância feminina.

Apesar dessas mudanças, Barros e Silva (2019), por outro lado, destacam que de forma mais geral as mulheres continuam sendo uma minoria no montante de bolsistas, tornando-se ainda mais rarefeita a presença delas nos níveis mais elevados. Esse cenário se vincularia a um conjunto de variáveis, dentre as quais as autoras destacam:

Enquanto os homens se titulam doutor e ingressam no sistema de bolsa produtividade mais jovens que as mulheres, tendo mais tempo para investir em suas produções científicas e progredir até o nível $1 \mathrm{~A}$ - chegando a $74 \%$ dos que estão nesse nível - as mulheres demoram mais para ingressar e se estabelecer no sistem a de bolsas do CN Pq. Portanto, a baix a representatividade feminina nos extratos mais elevados da carreira de bolsista $P Q$ pode estar associada a esse fenômeno e torna razoável a justificativa para a permanência das mulheres nos níveis iniciais. Esses achados apontam para as peculiaridades na trajetória feminina para alcançar cargos de maior nível hierárquico, levando aos questionamentos das práticas sociais que as naturalizam (Barros e Silva, 2019: 78).

Apesar disso, as autoras também destacam que pode ser observada certa mudança geracional com relação ao impacto das desigualdades de gênero na produção acadêmica. Entre as bolsistas mais jovens, que estão no nível inicial da bolsa, observa-se uma menor diferença em termos de produção acadêmica, o que poderia apontar também para a existência de mudanças na divisão do trabalho doméstico, algo que possui implicações diretas sobre a produção acadêmica.

De um modo geral, poderíamos dizer que há uma convergência nas pesquisas realizadas com relação ao reconhecimento do predomínio de homens entre os bolsistas PQ, algo que se acentua nos níveis mais elevados mas que tem apresentado alguma mudança nos últimos anos.

No que refere às questões metodológicas da presente investigação, para esse trabalho utilizamos uma amostra probabilística, de tipo aleatória simples, para uma população de 13 mil PQ. Consideramos um intervalo de confiança 3,91, com um nível de confiança de $95 \%$. O cálculo amostral resultou em uma 
amostra de 601 casos. A coleta dos 601 casos foi selecionada a partir da função sample do software R.

A partir da amostra selecionada dos $601 \mathrm{PQ}$, utilizamos uma técnica de web scrapping. Esta tem como função extrair rapidamente informações de sites e exportá-las para planilhas com o intuito de fazer análises e gerar insights para tomadas de decisão. Para tal, criamos uma função no software R para extração dos dados em xml dos currículos lattes dos bolsistas PQ. A função consiste na coleta automatizada de informações de um volume de currículos $n$, qualis das revistas e nota dos programas de pós-graduação.

A função retornava a uma lista com dataframes, que são tabelas ou uma estrutura de tipo matriz, em que cada coluna contém valores de uma variável e cada linha contém um conjunto de valores de cada coluna, com variáveis pertinentes à nossa investigação como: 1. informações gerais do pesquisador; 2 . conferências em que participou; 3 . livros publicados; 4. publicações de artigos; 5. orientações; 6. apresentações de trabalho; 7. atuação profissional; 8. bancas; 9. eventos; e 10. publicações midiáticas.

Para a manipulação dos dataframes foram utilizados os seguintes pacotes: dplyr, magrittr, rio. Demais variáveis foram coletadas através da consulta propriamente aos currículos lattes dos bolsistas e/ou por arquivos de consultas públicas disponíveis nos sites da CAPES (Plataforma Sucupira) e do CN Pq. Por fim, utilizamos o software Statistical Package for the Social Sciences - SPSS para tabulação e análise dos dados. Os nomes dos bolsistas (que não serão divulgados por questões éticas) foram colhidos na página web do $\mathrm{CN} \mathrm{Pq}^{4}$. O desenho da pesquisa foi de tipo interseccional/corte transversal, e a recolha dos dados ocorreu de janeiro a abril de 2020.

\section{Homens e mulheres entre os bolsistas PQ: apresentação e discussão dos dados}

Um primeiro dado geral que pode ser observado com relação à desigual distribuição de bolsistas PQ por sexo pode ser sintetizado no gráfico abaixo, que aponta que $63 \%$ dos bolsistas PQ são homens:

4 Lista de bolsistas disponível em: http://plsq11.cnpq.br/divulg/RESULTADO_PQ_102003.curso. 
Gráfico 1: Distribuição dos bolsistas PQ por sexo

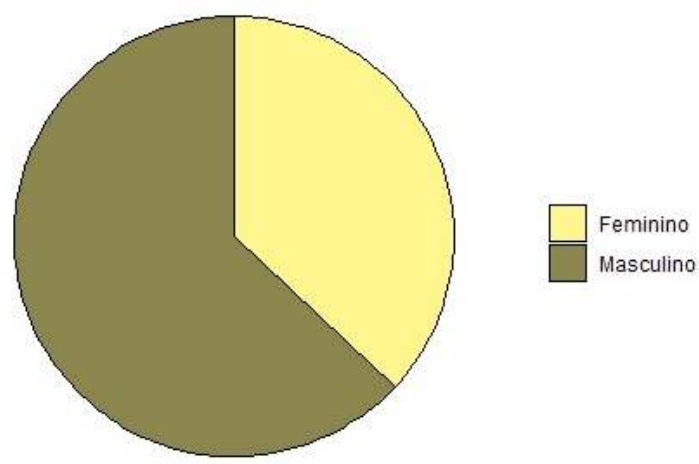

Fonte: Dados obtidos a partir da pesquisa (2020)

É importante considerar a existência de uma profunda estratificação dentro do próprio sistema de bolsas que, como já indicado, se organiza a partir de diferentes níveis de hierarquia. Essas bolsas são distribuídas da seguinte forma entre os diferentes níveis:

Gráfico 2: Distribuição por tipos de bolsa PQ

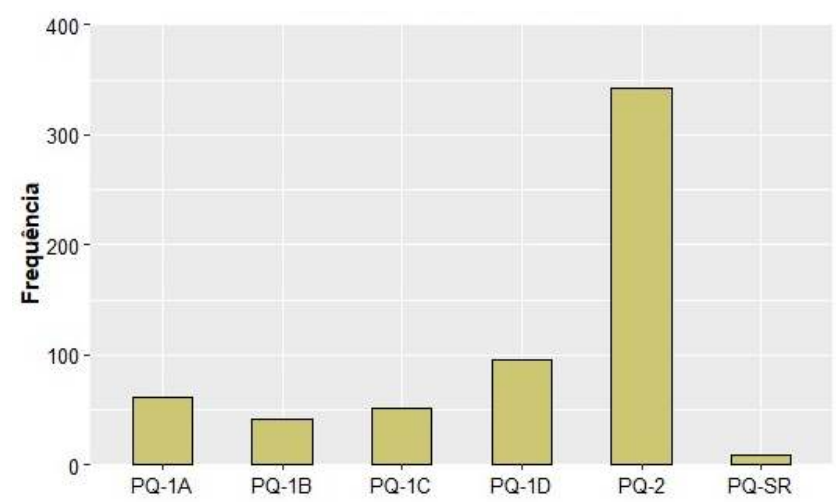

Fonte: Dados obtidos a partir da pesquisa (2020) 
Os bolsistas PQ-2 representam 57,1 \% dos casos; PQ1D são 15,8 \%; PQ1C são 8,7 \%; PQ1B são 6,8 \%; PQ1A são 10,1 \%; e PQ-Sr são 1,5 \% . Se considerarmos apenas a divisão existente entre o nível 2 e o nível 1 das bolsas PQ, temos que 60,9 \% dos bolsistas nível 2 são homens e 39,1\% mulheres. Entre os bolsistas nível 1 temos $65 \%$ de bolsistas homens e $35 \%$ de bolsistas mulheres, o que nos aponta uma tendência de menor presença das mulheres nos níveis mais elevados. Em todo o caso, esses dados também podem indicar que a desigual distribuição de bolsas PQ entre homens e mulheres é menor nas gerações mais recentes (majoritariamente com bolsistas nível 2), o que é um dado relevante, considerando-se que a maior parte dos bolsistas encontra-se neste nível.

Cabe-nos também examinar a distribuição entre homens e mulheres nesses diferentes graus de bolsa no nível 1. No nível 1D os homens são 62,1\% dos bolsistas e as mulheres $37,9 \%$; no nível 1C esses índices passam para 59,6\% e 40,4 \% ; no nível 1B são 65,8 \% e 34,2 \% ; no nível 1A são 73,7 \% e 26,3\%; e no nível SR são $88,8 \%$ e 11,2 \%, para homens e mulheres, respectivamente. Esses dados apontam para uma desigualdade mais profunda nos últimos níveis (PQ 1A e PQ SR). Estes são, justamente, os níveis nos quais as métricas quantitativas não seriam os únicos aspectos a serem avaliados para a classificação dos pesquisadores, segundo as normas do $\mathrm{CNPq}$, destacando-se também o papel de liderança científica em seu campo de atuação. Pode-se problematizar em que medida essa classificação dos pesquisadores como líderes em seus campos também é permeada pelas hierarquias de gênero, assim como pelas próprias condições de trabalho e desigualdade entre homens e mulheres, considerando as demais esferas da vida (trabalho doméstico).

Examinamos ainda essa distribuição com relação às diferentes grandes áreas, que também expressam uma hierarquia interna dentro desse campo. Assim sendo, as bolsas PQ estão distribuídas da seguinte forma entre as grandes áreas: Ciências Exatas e da Terra 19,6 \% ; Ciências Humanas 19,1 \%; Ciências Biológicas 15,6 \%; Engenharias 14,3 \%; Ciências da Saúde 10,5 \%; Ciências Sociais Aplicadas 7,8 \%; Ciências Agrárias 6,8 \%; e Linguística, Letras e Artes 6,2 \%. Passamos então para um exame interno dessas áreas com relação à distribuição entre homens e mulheres: 
Quadro 1: Distribuição por sexo dos bolsistas PQ nas grandes áreas

\begin{tabular}{|c|c|c|}
\hline Área & Homens & Mulheres \\
\hline Ciências Exatas e da Terra & $77,9 \%$ & $22,1 \%$ \\
\hline Ciências Humanas & $51,3 \%$ & $48,7 \%$ \\
\hline Ciências Biológicas & $54,2 \%$ & $45,8 \%$ \\
\hline Engenharias & $76,7 \%$ & $33,3 \%$ \\
\hline Ciências da Saúde & $61,9 \%$ & $38,1 \%$ \\
\hline Ciências Sociais Aplicadas & $63,8 \%$ & $36,2 \%$ \\
\hline Ciências Agrárias & $73,1 \%$ & $26,9 \%$ \\
\hline
\end{tabular}

Fonte: Dados obtidos a partir da pesquisa (2020)

Os dados expostos visibilizam que as mulheres apenas são a maior parte dos bolsistas PQ justamente na área com o menor número de bolsistas no total, e que a grande área com o maior número de bolsistas é também aquela onde há o mais expressivo contingente de homens. É possível inferir que a menor participação das mulheres entre os bolsistas PQ também se relaciona à sub-representação que elas possuem em determinadas áreas.

Encontramos diferença estatisticamente significativa entre as médias do índice de produção criado para esta investigação ${ }^{5}$ em relação às diferentes grandes áreas do CNPq (Anova; $\mathrm{p}=0,00$ ). Os índices encontrados foram os seguintes: Linguística, Letras e Artes 0,74; Ciências Humanas 0,82; Ciências

5 O índice de produção acadêmica geral dos PQsbrasileiros foi formado com as seguintes variáveis: 1. Quantidade de apresentação de trabalhos em congressos, palestras, seminários, etc.; 2. Quantidade de textos apresentados em congressos, palestras, seminários, etc.; 3. Quantidade de livros publicados desde 2015; 4. Quantidade total de artigos publicados em revistas desde 2015; 5. Quantidade total de artigos publicados avaliados pelo Webqualis da CAPES; 6. Total de artigos publicados de Estrato Superior avaliados pelo Webqualis da CAPES; 7. Quantidade de publicações midiáticas não especializadas (TV, jornais, etc.); 8. Total de orientações concluídas desde 2015; 9. Quantidade de participações em bancas de doutorado desde 2015; 10. Quantidade de participações em bancas de mestrado desde 2015; 11. Quantidade de participações em bancas de graduação desde 2015; 12. Quantidade de participações em bancas de especialização desde 2015; e;13. Quantidade de participação em eventos desde 2015. A variável original, o índice criado, apresentou-se fora dos padrões de normalidade, no que optamos por utilizar o índice desta pesquisa a partir de uma variável criada com correção logarítmica (LG10). As medidas de tendência centrais básicas do índice criado a partir das 13 variáveis e com 601 casos são: média de produção acadêmica geral/LG10 (0,919); ;esvio padrão $(0,29)$ e amplitude (de -0,20 a 1,92). Nota-se que o índice foi criado sem nenhum tipo de ponderação, ou seja, somente a partir de valores brutos, o que equivale a dizer que não foi realizado nenhum tipo de decisão sobre quais aspectos teoricamente pesariam mais na questão da produção. Optamos por utilizar os dados brutos justamente para que, na análise, pudéssemos comparar as formas de produção sem aspectos valorativos prévios que pudessem considerar umas variáveis mais interessantes do que outras no entendimento da produção. 
Sociais Aplicadas 0,84; Ciências Exatas e da Terra 0,85; Engenharias 0,95; Ciências Biológicas 0,99; Ciências Agrárias 1,00; Ciências da Saúde 1,15. Também quando examinamos o índice de produção no qualis superior das áreas de avaliação dos pesquisadores encontramos diferença estatisticamente significativa entre as médias de publicações (Anova; $\mathrm{p}=0,00$ ), sendo as seguintes médias nas grandes áreas: Ciências Humanas 0,50; Linguística, Letras e Artes 0,51; Ciências Sociais Aplicadas 0,54; Engenharias 0,95; Ciências Exatas e da Terra 0,98; Ciências Biológicas 1,04; Ciências Agrárias 1,13; Ciências da Saúde 1,23. Observa-se que as duas áreas com maior participação feminina foram as que apresentaram também as menores médias. Deve-se ressaltar, no entanto, que as grandes áreas possuem lógicas diferentes de produção, havendo uma tradição mais recorrente de trabalhos sem co-autoria nas Letras e Ciências Humanas, assim como menor financiamento para pesquisa.

Também nos diferentes balanços que são realizados acerca do perfil dos bolsistas PQ, considerando as mais diversas áreas do conhecimento, recorrentemente é destacado a desigual distribuição de bolsas entre as diferentes regiões do País. Esse processo se relaciona à concentração histórica de programas de pós-graduação na região sudeste do Brasil, assim como a existência de um maior volume de recursos disponível para pesquisa através das fundações locais de amparo à pesquisa. Programas de pós-graduação localizados em regiões com mais recursos para pesquisa tendem a encontrar melhores condições para realizar suas publicações, o que tende a reforçar as desigualdades regionais. Em nossa amostra encontramos o seguinte cenário: 57,6 \% dos bolsistas são do Sudeste, $21,1 \%$ do Sul, 15,8 \% do Nordeste; $4 \%$ do Centro-Oeste e 1,3 $\%$ do Norte.

Gráfico 3: Distribuição dos bolsistas PQ por região do Brasil

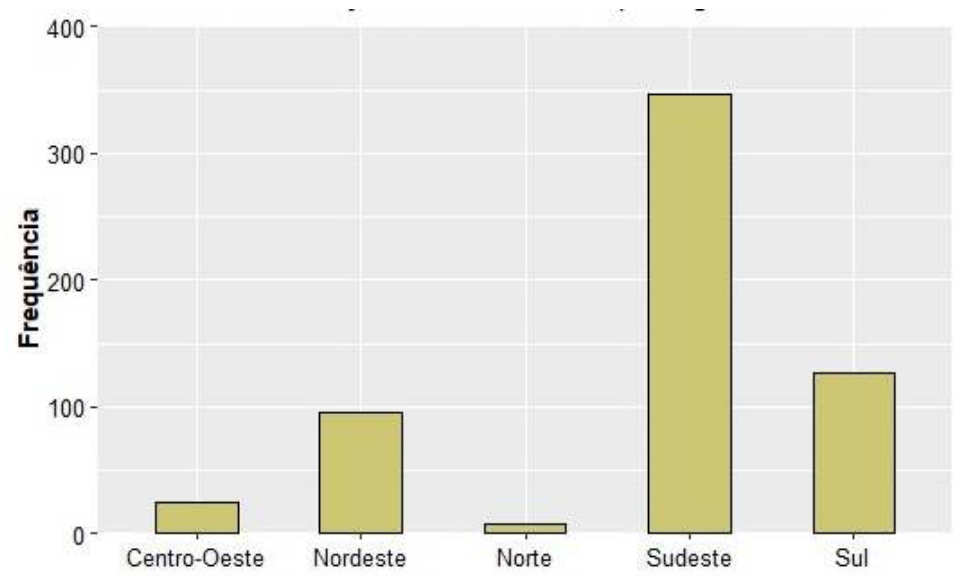

Fonte: Dados obtidos a partir da pesquisa (2020) 
Ainda sobre as desigualdades regionais da ciência no Brasil, dentro dessas regiões temos os seguintes estados com as maiores concentrações de bolsistas: São Paulo (SP) com 31,8 \%; Rio de Janeiro (RJ) com 15,5\%; Rio Grande do Sul (RS) com $12 \%$; Minas Gerais (MG) com $10 \%$; e Paraná (PR) com 6,5\%.

Examinando especificamente esses estados, encontramos o seguinte cenário em relação à distribuição de homens e mulheres entre seus bolsistas PQ: em SP 60,7 \% dos bolsistas são homens e 39,3 \% são mulheres; no RJ, 59,1 $\%$ e $40,9 \%$; no RS, $61,1 \%$ e 38,9 \%; em MG, 68,3\% e 31,7\%; no PR, $61,5 \%$ e $38,5 \%$, respectivamente. Isso significa que também nos estados com maior número de bolsistas encontramos um cenário semelhante ao resto do País, em termos de desigualdades de distribuição das bolsas PQ entre homens e mulheres.

Considerando-se a distribuição dos pesquisadores por notas dos programas de pós-graduação (numa escala de 3 a $7^{6}$ ), temos o seguinte cenário: 26,8 $\%$ em programas nota $4 ; 26 \%$ em programas nota $5 ; 21,1 \%$ em programas nota $7 ; 18,6 \%$ em programas nota 6 e; 3,3\% em programas nota 3 . É interessante perceber que, apesar de a maior parte dos programas de pós-graduação no Brasil serem de notas 3 (1478) e 4 (1530), e serem minoria os programas de notas 6 (307) e 7 (185), observa-se que, proporcionalmente, ocorre uma maior concentração de bolsistas nesses dois níveis mais elevados, reforçando a dificuldade de os programas de nota baixa ascenderem, posto que ter bolsistas PQ no corpo docente favorece também a avaliação dos programas. Entre os bolsistas PQ vinculados aos programas com notas mais altas, temos o seguinte cenário: 62,2 \% dos bolsistas PQ vinculados a programas nota 7 são homens, ao passo que $37,8 \%$ são mulheres. Esses números passam, respectivamente, para $66 \%$ e $33 \%$ nos programas nota $6 ; 62,8 \%$ e $37,2 \%$ nos programas nota $5 ; 60,2 \%$ e $39,8 \%$ nos programas nota 4 e; $80 \%$ e $20 \%$ nos programas nota 3 . Como podemos observar, os homens constituem a maioria dos bolsistas PQ nos programas situados nas diferentes faixas de classificação em termos de notas.

Chama a atenção também o fato de que, apesar de a grande maioria dos bolsistas PQ serem professores de Instituições de Ensino Superior Públicas $(91,5 \%)$, e apenas uma pequena minoria estar vinculada às instituições privadas e ao terceiro setor $(9,5 \%)$, há diferenças também entre homens e mulheres nesse quesito. Entre as mulheres, 88,2 \% são professoras em IES públicas, índice que sobe para 93,4 \%, o que tende a apontar para o fato de que entre os bolsistas PQ homens são mais recorrentes vínculos empregatícios mais estáveis no ensino superior.

6 No Brasil, a CAPES avalia os programas de pós-graduação em uma escala que vai de 0 a 7. Podem funcionar com cursos de mestrado apenas os programas avaliados a partir da nota 3 . Somente programas com nota igual ou superior a 4 podem se candidatar a oferta recursos de doutoramento. 
A relação entre as desigualdades de gênero e o trabalho acadêmico também podem ser inferidas através do dado referente ao tempo de conclusão do doutorado. De acordo com nossa análise, existe diferença estatisticamente significativa entre bolsistas PQ homens e mulheres a respeito do tempo que levaram para concluir o curso de doutorado (Teste T, p=0,00). Em média, as mulheres demoraram 4,3 anos para concluir o doutorado, e os homens 3,8 anos.

É importante dizer, no entanto, que, ao aplicarmos o teste T, encontramos uma média de produtividade, de acordo com o índice de produtividade criado, igual a 0,93 entre homens e 0,90 entre mulheres, sem sig $(\mathrm{p}=0,28)$. Sendo assim, não existe diferença estatisticamente significativa entre as médias de produção de homens e mulheres. O mesmo pode ser dito com relação à produção de artigos qualificados no qualis superior pela área na qual os pesquisadores são vinculados. Ao aplicarmos o teste T, encontramos uma média de publicação em qualis superior entre homens de 0,92 e entre mulheres de 0,87 , sem sig $(\mathrm{p}=0,22)$. Assim sendo, não existe diferença estatisticamente significativa entreCapes dee mulheres nas médias de publicação em revistas de qualis superior no Webqualis da CAPES.

Coadunando com uma série de análises já realizadas em torno da relação entre gênero e atividade acadêmica, observamos no Brasil uma predominância masculina nas diversas áreas do conhecimento, regiões e notas de programas. Todavia, também vale destacar que os níveis mais elevados de bolsas PQ, assim como as áreas que contam com um maior número de bolsistas, são áreas com uma composição ainda maior de homens. Podemos inferir, portanto, que a sub-representação das mulheres em áreas que recebem mais bolsas PQ tende a reforçar essa desigual distribuição dos recursos do CN Pq.

Ao analisarmos especificamente a distribuição de homens e mulheres entre os bolsistas nível 1A e SR, observamos que são nesses níveis que encontramos proporcionalmente menos mulheres, e também é nesses níveis que a dimensão quantitativa da produção acadêmica apresenta um peso mais significativo. Segundo as normas do CNPq, esses níveis são reservados para as lideranças acadêmicas em seus respectivos campos. As discrepâncias de gênero apontam para a presença de um conjunto de obstáculos ao longo da trajetória feminina que dificulta que elas cheguem ao topo do reconhecimento acadêmico. Estes obstáculos são materializados por meio de uma sucessão de dificuldades respaldadas por práticas sociais pouco explícitas com as quais as mulheres precisam lidar (Barros e Silva, 2019). Poderíamos inferir que tais questões encontram-se mais explicitadas nos níveis mais altos da hierarquia dos bolsistas PQ. Neste sentido, são interessantes as questões trazidas por Lima (2013), ao analisar as trajetórias das pesquisas no campo da física, de modo que a autora chama a atenção para algumas questões que surgiram em sua pesquisa e que, apesar das particularidades do campo acadêmico da física, poderiam ser encontradas também em outros campos: 
(...) o entendimento isolado de suas trajetórias profissionais (obstáculos e superações); o relato de terem suas habilidades e competências postas à prova ao ocuparem determinadas posições na carreira; a dificuldade em conciliar maternidade e família; a pressão do meio em adequar seu comportamento à cultura androcêntrica, que define critérios para atuação e ascensão na carreira com referência ao padrão masculino hegemônico; e o relato de discriminações implícitas e estruturais. No entanto, também destaca-se que alguns obstáculos são específicos do meio acadêmico e, mais propriamente, da área da física. $O$ discurso meritocrático, ainda que presente em outros ambientes profissionais, é central no meio acadêmico. (Lima, 2013: 898)

Se compararmos com levantamentos semelhantes realizados anteriormente, como aquele feito por Guedes, Azevedo e Ferreira (2015), observamos a consolidação de algumas tendências. As Ciências Humanas, apesar de serem uma das grandes áreas com maior presença de mulheres, apresentam em nossa amostra mais pesquisadores, o que pode indicar uma tendência de aumento da presença masculina em campos historicamente ocupados por mulheres. Ademais, devem-se considerar ainda as hierarquias existentes dentro das próprias áreas. Leite e Rocha Neto (2017), por exemplo, ao analisarem o perfil dos bolsistas PQ em educação, apontam para uma predominância massiva de pesquisadoras nesse quadro; porém, indicam também que essa presença diminui nos níveis mais elevados.

Chama a atenção o fato de que, apesar de as mulheres terem demorado mais anos para realizarem seus doutorados (com diferença estatisticamente significativa), o que pode se relacionar principalmente à divisão assimétrica do trabalho doméstico, sobretudo nas gerações mais velhas de pesquisadoras (Barros e Silva, 2019), não houve diferença estatisticamente significativa em termos de produção acadêmica, incluindo no qualis superior da área de avaliação dos pesquisadores. Isso pode significar que essas pesquisadoras encontraram condições mais adversas para a consolidação de suas carreiras acadêmicas durante seu processo formativo, sendo paulatinamente superadas, ainda que não completamente. Esse dado tende a confluir com as análises que enfatizam o bom desempenho das mulheres nas carreiras universitárias (Marrero, 2006).

\section{Conclusão}

Considerando-se o papel que a distribuição das bolsas PQ desempenha no estabelecimento de hierarquias no campo acadêmico brasileiro, sendo um dos principais signos do reconhecimento da comunidade de pares, a análise de sua dinâmica e distribuição entre homens e mulheres constitui um dos mais 
relevantes esforços para a compreensão das desigualdades de gênero neste campo. Observando-se os dados coletados, torna-se evidente que, para além das normas oficiais, o campo se estrutura também a partir de normas socialmente implícitas que em muitos casos implicam no aprofundamento das desigualdades.

Os dados que apresentamos apontam para essa sub-representação das mulheres nos diferentes cenários de distribuição das bolsas PQ (nível da bolsa, grande área científica, região do Brasil, notas dos programas de pós-graduação aos quais os PQ estão vinculados). Existe uma melhor participação feminina justamente naqueles programas com menos bolsas e com menor produção acadêmica. Isso pode ser interpretado como uma estrutura que reforça a exclusão das mulheres de áreas que obtêm mais recursos para pesquisa e divulgação científicas, especialmente ante um novo cenário que vem se desenhando no $\mathrm{CNPq}$ de delimitação de áreas prioritárias.

A menor presença de mulheres nos níveis mais elevados das bolsas PQ (1 A e SR) pode apontar tanto para um reforço das hierarquias de gênero na academia, como para uma mudança geracional em curso. Um acompanhamento longitudinal desses agentes poderia dar pistas sobre o tempo necessário para homens e mulheres passarem de PQ2 (nível inicial) para PQ1. Como já assinalado, o nível 1 da bolsa de produtividade implica no acesso não apenas a bens materiais (a taxa de bancada que acompanha a bolsa, além de acesso a editais específicos de financiamento), como também a bens simbólicos, como a possibilidade de compor os comitês de assessoramento do CN Pq.

Os obstáculos observados nesta investigação podem ser inferidos a partir do maior tempo para a realização do doutorado e da menor proporção de pesquisadoras vinculadas a IES públicas, bem como da não existência de diferença estatisticamente significativa na produção de homens e mulheres, incluindo-se a publicação de artigos no qualis superior. Este cenário indica também a existência de condições relativamente favoráveis para a consolidação das carreiras dessas pesquisadoras a médio prazo. A maior inserção em áreas como Letras e Ciências Humanas também deve ser considerada nesse processo, uma vez que são áreas com acesso a menores financiamentos e com uma lógica de produção mais autoral, sendo menos recorrente a produção acadêmica em co-autoria ou em equipes.

É necessário dizer que esse estudo traz como contributo para futuras investigações sobre gênero e ciência a crítica de que existe uma lógica de reprodução das desigualdades de gênero que é estudada na academia, mas que precisa sair de si, sair dos intramuros acadêmicos, sair do plano da reflexão para a ação, posto que demonstramos, assim como outros estudos o fazem, que as desigualdades de gênero estudadas pela academia também se dão dentro desta. Nossa investigação pode suscitar questões importantes a serem analisadas por trabalhos futuros, considerando outros marcadores como geração e raça, ou ainda o cruzamento de outros dados que parecem ter um peso importante na 
determinação da carreira investigativa. Afinal, quão mais provável é para um pesquisador homem localizado na região sudeste do Brasil se tornar bolsista PQ em relação a uma pesquisadora mulher localizada na região norte, por exemplo? Esta questão fica em aberto, devendo ser respondida por investigações futuras.

\section{Referências bibliográficas}

BARBOSA, Maria Ligia (2015), "Destinos, escolhas e a democratização do Ensino Superior", Política \& Sociedade, 14 (31), 256-282.

BARBOSA, Maria Ligia (2014), Ensino Superior: Expansão e Democratização, Rio de Janeiro, Editora 7 Letras.

BARBOSA, Maria Ligia, GANDIN, Luís A. (2020), "Sociologia da educação brasileira: diversidade e qualidade", Revista Brasileira de Informações Bibliográficas em Ciências Sociais - BIB, (91), 1-38.

BARROS, Suzane C. V., SILVA, Luciana M. C. (2019), "Desenvolvimento na carreira de bolsistas produtividade: uma análise de gênero", A rquivos Brasileiros de Psicologia, 71 (2), 68-83.

CONSELHO NACIONAL DE DESEN VOLVIMENTO CIENTÍFICO E TECNOLÓGICO (2015), Bolsas Individuais no País, RN 028/2015. Disponível em: cnpq.br/web/guest/view/-/journal_content/56_ INSTANCE_0oED/10157/2958271. Acesso em 12 de agosto de 2020.

FERREIRA, Márcia Ondina Vieira, CORONEL, Márcia Cristiane V. K. (2017), "Sobre a legitimação do campo do gênero na ANPEd", Educação e Pesquisa, 43 (3), 815-832.

FREITAS, Lucas B., LUZ, Nanci S. (2017), "Gênero, Ciência e Tecnologia: estado da arte a partir de periódicos de gênero", Cadernos Pagu, (49), 1-26.

GROSSI, Márcia G. R., BORJA, Shirley D. B., LOPES, Aline M., ANDALÉCIO, Aleixina M. L. (2016), “As mulheres praticando ciência no Brasil”, Revista Estudos Feministas, 24 (1), 11-30.

GUEDES, Moema C., AZEVEDO, Nara, FERREIRA, Luiz O. (2015), “A produtividade científica tem sexo? Um estudo sobre bolsistas de produtividade do CN Pq", Cadernos Pagu, (45), 367-399.

LEITE, Anderson Cleiton Fernandes, ROCH A NETO, Ivan (2017), "Perfil dos bolsistas de produtividade em pesquisa do CN Pq em Educação", Revista Brasileira de Ensino Superior, 3 (4), 97-112.

LIM A, Betina S. (2013), "O labirinto de cristal: As trajetórias das cientistas na Física", Revista Estudos Feministas, 21 (3), 883-903.

LIM A, Betina S., COSTA, Maria C. (2016), "Gênero, ciências e tecnologias: caminhos percorridos e novos desafios", Cadernos Pagu, (48), 120-158.

LOPES, Maria Margaret (1998), “'Aventureiras' nas ciências: Refletindo sobre gênero e história das ciências naturais no Brasil”, Cadernos Pagu, (10), 345-368.

MARRERO, Adriana (2006), "El asalto femenino a la universidad: un caso para la discusión de los efectos reproductivos del sistema educativo en relación al género", Revista A rgentina de Sociología, 4 (7), 47-69.

MARTINS, Carlos Benedito (2018), “As origens da pós-graduação nacional”, Revista Brasileira de Sociologia, 6 (13), 9-26. 
NEVES, Clarissa Eckert Baeta, RAIZER, Leandro; FACHINETTO, Rochele Fellini (2007), "Acesso, expansão e eqüidade na educação superior: novos desafios para a política educacional brasileira", Sociologias, (17), 124-157.

OGBOGU, Christiana O. (2011), "Gender Inequality in Academia: evidences from Nigeria", Contemporary Issues in Education Research, 4 (9), 1-8.

OLIVEIR A, Amurabi, SILVA, Camila Ferreira (2020), "The Sociology of Education in Brazil Today", RASE - Revista de Sociología de la Educación, 13 (1), 39-54.

OLSEN, Deborah, MAPLE, Sue, STAGE, Frances (1995), "Women and minority faculty job satisfaction", Higher Education, 66 (3), 267-292.

POOLE, Millicent, BORN HOLT, Laurel, SUMMERS, Fiona (1997), “An international study of the gendered nature of academic work: Some cross-cultural explorations", Higher Education, 34 (3), 373-396.

PROBERT, Belinda (2005), “'I just couldn't fit it in': Gender and unequal outcomes in academic careers", Gender, Work and O rganization, 12 (1), 50-72.

SARSONS, Heather (2017), "Recognition for Group Work: Gender Differences in Academia", American Economic Review, 107 (5), 141-45.

SCHWARTZMAN, Simon (1979), Formação da Comunidade Científica no Brasil, Rio de Janeiro e São Paulo, FIN EP e Companhia Editora Nacional.

TOREN, Nina (1993), "The temporal dimension of gender inequality in academia", Higher Education, (25), 439-455.

WEINER, Jacques, VIEIRA, Paulo (2013), Avaliação de bolsas de produtividade em pesquisa do $\mathrm{CNPq}$ e medidas bibliométricas: correlações para todas as grandes áreas, Perspectivas em Ciência da Informação, 18 (2), 60-78.

WEISSH AAR, Katherine (2017), "Publish and Perish? An Assessment of Gender Gaps in Promotion to Tenure in Academia", Social Forces, 96 (2), 529-560. 